\title{
Gynomonoecy in a mycoheterotrophic orchid Eulophia zollingeri with autonomous selfing hermaphroditic flowers and putatively outcrossing female flowers
}

\author{
Kenji Suetsugu ${ }^{\text {Corresp. } 1}$ \\ ${ }^{1}$ Department of Biology, Graduate School of Science, Kobe University, Kobe, Japan \\ Corresponding Author: Kenji Suetsugu \\ Email address: kenji.suetsugu@gmail.com
}

Most orchid species exhibit an extreme case of hermaphroditism, owing to the fusion of male and female organs into a gynostemium. E xceptions to this rule have only been reported from the subtribes Catasetinae and Satyriinae. Here, I report an additional orchidaceous example whose flowers are not always hermaphroditic. In Japanese populations of Eulophia zollingeri (Rchb.f.) J.J.Sm, a widespread Asian and Oceanian orchid, some flowers possess both the anther (i.e., anther cap and pollinaria) and stigma, whereas others possess only the stigma. Therefore, pollination experiments, an investigation of floral morphology and observations of floral visitors were conducted to understand the reproductive biology of $E$. zollingeri in Miyazaki Prefecture, Japan. It was confirmed that $E$. zollingeri studied here pos sesses a gynomonoecious reproductive system, a sexual system in which a single plant has both female flowers and hermaphroditic flowers. In addition, hermaphroditic flowers often $\mathrm{p}$ ossessed an effective self-pollination system while female flowers could avoid autogamy but suffered from severe pollinator limitation, due to a lack of agamospermy and low insect-mediated pollination. The present study represents the first documented example of gynomonoecy within Orchidaceae. Gynomonoecy in $E$. zollingeri may be maintained by the tradeoff in reproductive traits between female flowers (with low fruit set but potential outcrossing benefits) and hermaphroditic flowers (with high fruit set but inbreeding depression in selfed offspring). This mixed mating is probably important in mycoheterotrophic $E$. zollingeri because it occurs in shaded forest understorey with a paucity of pollinators. 


\section{Gynomonoecy in a mycoheterotrophic orchid}

\section{Eulophia zollingeri with autonomous selfing}

\section{3 hermaphroditic flowers and putatively outcrossing}

\section{4 female flowers}

5

6 Kenji Suetsugu ${ }^{1}$

7

Kenji Suetsugu ${ }^{1}$

\section{Abstract}

${ }^{1}$ Department of Biology, Graduate School of Science, Kobe University, Kobe, Japan

Corresponding Author:

Department of Biology, Graduate School of Science, Kobe University, 1-1 Rokkodai, Nada-ku, Kobe, 657-8501, Japan

Email address: kenji.suetsugu@gmail.com

Most orchid species exhibit an extreme case of hermaphroditism, owing to the fusion of male and female organs into a gynostemium. Exceptions to this rule have only been reported from the subtribes Catasetinae and Satyriinae. Here, I report an additional orchidaceous example whose flowers are not always hermaphroditic. In Japanese populations of Eulophia zollingeri (Rchb.f.) J.J.Sm, a widespread Asian and Oceanian orchid, some flowers possess both the anther (i.e., anther cap and pollinaria) and stigma, whereas others possess only the stigma. Therefore, pollination experiments, an investigation of floral morphology and observations of floral visitors were conducted to understand the reproductive biology of E. zollingeri in Miyazaki Prefecture, Japan. It was confirmed that E. zollingeri studied here possesses a gynomonoecious reproductive system, a sexual system in which a single plant has both female flowers and hermaphroditic flowers. In addition, hermaphroditic flowers often possessed an effective self-pollination system while female flowers could avoid autogamy but suffered from severe pollinator limitation, due to a lack of agamospermy and low insect-mediated pollination. The present study represents the 
30 first documented example of gynomonoecy within Orchidaceae. Gynomonoecy in E. zollingeri

\section{Introduction} pollinators. (Barrett, 2010). may be maintained by the tradeoff in reproductive traits between female flowers (with low fruit set but potential outcrossing benefits) and hermaphroditic flowers (with high fruit set but inbreeding depression in selfed offspring). This mixed mating is probably important in mycoheterotrophic E. zollingeri because it occurs in shaded forest understorey with a paucity of

Keywords autogamy, breeding system, geographic variation, gynomonoecy, mixed mating, mycoheterotrophy, outcrossing, reproductive assurance, self-pollination

The Orchidaceae are one of the largest and most morphologically diverse families of land plants, and include more than 28,000 species classified into 760 genera (Christenhusz \& Byng, 2016). Variations in its floral characteristics and their effects on reproductive success have long intrigued botanists since the time of Darwin (Inoue, 1986; Nilsson, 1988; Sletvold \& Gren, 2011). However, it is noteworthy that while the vast majority of orchid species produce only hermaphroditic flowers (i.e., flowers that possess both male and female reproductive organs; Pannell, 2009), a variety of sexual polymorphisms (the co-occurrence of morphologically distinct sex phenotypes within the same species) can be found in flowering plants as a whole

In fact, almost all orchid species exhibit an extreme case of hermaphroditism, owing to the fusion of male and female organs into a gynostemium (Rudall \& Bateman, 2002). Exceptions to this rule have only been reported from the subtribes, Catasetinae and Satyriinae (Pannell, 2009; Romero \& Nelson, 1986; Huang et al., 2009). More specifically, within Catasetinae, the members of Catasetum Rich. ex Kunth and Cycnoches Lindl. typically exhibit dioecy (i.e., unisexual individuals). In the dioecious Catasetum, male flowers forcibly attach a large pollinarium onto euglossine bees, and the bees subsequently avoid flowers with the same appearance (Romero \& Nelson, 1986). Therefore, Catasetum populations are sexually dimorphic, and the agitated pollinators bearing their pollinia move away from the male flowers to the morphologically different female flowers (Romero \& Nelson, 1986). In addition, within Satyriinae, Satyrium ciliatum Lindl. has been reported to produce both hermaphroditic and 
61 female individuals (i.e., gynodioecy; Huang et al., 2009). Female individuals of S. ciliatum can

62 avoid pollen limitation for seed production and can be maintained in populations that experience

63 high levels of pollen limitation, because females, via facultative parthenogenesis, can produce

64 more seeds than do hermaphrodites (Huang et al., 2009). These observations helped elucidate the 65 unusual maintenance of gender dimorphism in orchids (Pannell, 2009).

Here, I report an additional orchidaceous example whose flowers are not always

67 hermaphroditic. In a Japanese population of Eulophia zollingeri (Rchb.f.) J.J.Sm, a widespread

68 Asian and Oceanian orchid, some flowers possess both the anther (i.e., anther cap and pollinaria)

69

70

71

72

73

74

75

76

77

78

79

80

81

82

83

84

85

86

87

88

89

90

91

and stigma, whereas others possess only the stigma (Fig. 1). It is unlikely that the absence of anther cap and pollinaria is the result of removal by floral visitors, because careful dissection revealed that they were already absent before anthesis. Such a sexual system, in which plants have both female and hermaphroditic flowers co-occurring within the same plants, is called gynomonoecy. Compared with andromonoecy (male and hermaphroditic flowers within one plant) and monoecy (separate male and female flowers on the same plant), gynomonoecy remains a poorly studied sexual system, even though it occurs in $2.8-4.7 \%$ of flowering plants in at least 15 plant families (Lu \& Huang, 2006; Yampolsky \& Yampolsky, 1922).

In fact, while several hypotheses have been proposed, the adaptive significance of gynomonoecy remains largely unknown. First, the presence of the two flower types may permit flexible allocation of resources to female and male reproductive functions in response to environmental conditions (Charnov \& Bull, 1977; Lloyd, 1979). Second, female flowers may promote outcrossing more than hermaphroditic flowers (Marshall \& Abbott, 1984). Third, due to the lack of evidence for the above two hypotheses, it has been proposed that female flowers may boost attractiveness to pollinators (Bertin \& Kerwin, 1998). Finally, female flowers may be favored because hermaphroditic flowers, which are more attractive in gynomonoecious plants, are more susceptible to florivory (Bertin, Connors \& Kleinman, 2010; Zhang, Xie \& Du, 2012). Meanwhile, current understanding of the adaptive advantages of gynomonoecy is largely limited to the Asteraceae and to a few species that have been investigated in other families (Davis \& Delph, 2005; Mamut et al., 2014).

Therefore, I investigated the reproductive biology of a Japanese E. zollingeri population, which potentially represents the first documentation of gynomonoecy within Orchidaceae, to understand the ecological significance of the different reproductive strategies of the two floral

Peer) reviewing PDF | (2020:07:51078:2:1:NEW 26 Sep 2020) 
92 morphs within one individual. Autonomous self-pollination has been suggested to be favorable

93 for mycoheterotrophic plants, as they are restricted to dark shaded forest understorey with a 94 paucity of pollinators (Leake, 1994; Zhou et al., 2012; Suetsugu, 2013a, 2015). However, the

95 Red Queen hypothesis suggests that sexual reproduction is important in a coevolutionary arms 96 race between a parasite and host (Ladle, 1992), such as between mycoheterotrophic plants and 97 fungal hosts. Intriguingly, Zhang et al. (2014) revealed Chinese populations of E. zollingeri to be 98 hermaphroditic, non-rewarding, self-compatible, and dependent on the halictid bee Nomia 99 viridicinctula Cockerell for pollination through food deception. Although those Chinese 100 populations consequently experienced strong pollinator limitation, especially in forest 101 understorey populations (Zhang et al., 2014), my preliminary investigation revealed that 102 Japanese populations consistently exhibit high fruit set even under the shaded forest understorey. 103 Therefore, it is possible that the Japanese hermaphroditic flowers are capable of autonomous 104 selfing, providing reproductive assurance, while female flowers enhance outcrossing However, it should also be noted that the "female" E. zollingeri flowers can be sterile without not only the male but also the female reproductive function. In fact, in Catasetum

107 108 109 species that produce male and female flowers, intermediate flowers, which are sterile, have also been found (Romero, 1992). Therefore, I first investigated whether the "female" flowers of $E$. zollingeri possess female reproductive functions. After that, I conducted additional pollination experiments, investigations of floral morphology, and observations of floral visitors to determine the potential for reproductive assurance provided by autonomous selfing in female flowers and outcrossing via pollinator visitation in both hermaphroditic and female flowers.

\section{Materials \& Methods}

Eulophia zollingeri is a mycoheterotrophic orchid distributed from India and Southeast Asia to New Guinea and Australia (Ogura-Tsujita \& Yukawa, 2008; Suetsugu \& Mita, 2019; Suetsugu, Matsubayashi \& Tayasu, 2020). The behavior of floral visitors in Miyazaki City, Miyazaki Prefecture, Japan, was monitored during the peak flowering period (early to mid-July), in 2016 and 2017. Direct observations were made for ca. $30 \mathrm{~h}$ in total, during the peak of diurnal insect activity (09:00-17:00). The behavior of potential visitors was observed by walking around the study site, sitting next to flower patches, or hiding in the vegetation near flower clusters (within 1-2 m). In addition, artificial cross-pollination was performed in the same population in July 2016, 
123 by transferring pollinaria from different individuals to the stigmas of female flowers (five 124 inflorescences, 10 flowers).

125 After confirming with the cross-pollination experiment that both hermaphroditic and female 126 flowers produce fruits, additional pollination experiments were conducted in early July 2017. 127 Flowers were either (i) manually cross-pollinated by transferring pollinaria to the stigmas of 128 different individuals (five inflorescences, 10 each of female and hermaphroditic flowers); (ii) 129 manually geitonogamous-pollinated by transferring pollinaria to the stigmas of the different 130 flowers within the same individuals for female flowers and of the same flowers for hermaphroditic 131 flowers (five inflorescences, 10 each of female and hermaphroditic flowers); (iii) enclosed in mesh 132 bags to exclude floral visitors and test for autonomous self-pollination (five inflorescences, 10 133 hermaphroditic flowers); or (iv) left unmanipulated, in order to monitor fruit set under natural 134 conditions (seven inflorescences, 19 female flowers and 21 hermaphroditic flowers). In addition, 135 the relative position of female and hermaphroditic flowers on the racemes was determined in 12 136 inflorescences. Furthermore, to compare flower size between female and hermaphroditic flowers 137 in gynomonoecious individuals, we measured the length of the dorsal sepal, lateral sepal, lateral 138 petal, and lip of 10 plants using digital calipers to $0.1 \mathrm{~mm}$ in early July 2017. Finally, the 139 distribution of female flowers was checked in 12 inflorescences in early July 2017. After dividing 140 each inflorescence into distal and proximal halves, the data were tested using the Mann141 Whitney $U$-test to investigate whether female flowers tended to be in the distal or basal part of the 142 inflorescence.

143 Three to four months after manual pollination, all the mature but non-dehisced fruits 144 capsules were collected. After the fruits were silica-dried, I weighed the total mass of dry seeds 145 freed from each capsule to the nearest $0.1 \mathrm{mg}$. All the seeds from each plant were then mixed, and 146100 randomly selected seeds from each plant were examined under a dissecting microscope to 147 determine presence of an embryo. The effects of pollination treatment on fruit set were tested using 148 Fisher's exact test. In addition, after confirming that the datasets were normally distributed using 149 Levene's test, the effects of pollination treatment on the seed mass, and the proportion of seeds 150 with an embryo were tested using ANOVA, followed by Fisher's multiple comparisons test.

\section{Results}


153 Despite conducting ca. $30 \mathrm{~h}$ of field observations, few insects were observed visiting the $E$.

154 zollingeri flowers. Several dipteran visitors, such as the agromyzid fly Japanagromyza

155 tokunagai, occasionally landed on the flowers. However, none of these visitors were observed to

156 remove or deposit pollinaria. The length of the dorsal sepal, lateral sepal, lateral petal, and lip

157 were not significantly different between female and hermaphroditic flowers (Table 1). In

158 addition, there are marginally significant differences in the number of female flowers between

159 the distal half $(1.2 \pm 1.1$; mean $\pm \mathrm{SD})$ and the proximal half $(3.3 \pm 3.4 ; P=0.06)$ of the

160 inflorescence.

161 More than half (6/10) of the female flowers subsequently developed fruit capsules that

162 contained seeds with an embryo through artificial cross-pollination in 2016, thereby

163 demonstrating their female function and confirming the gynomonoecy of the species. The results

164 are stable at least in the investigated site, because similar results were obtained in 2017 (Table 2).

165 The detailed pollination experiments showed that the bagged female flowers failed to develop

166 fruits autonomously, excluding the possibility of agamospermy, while comparable fruit set ratio

167 was also obtained in open, bagged, manual geitonogamous and allogamous hermaphroditic

168 flowers. Therefore, the hermaphroditic flowers are capable of outbreeding, but self-compatible

169 and not pollinator-limited for fruit set under natural condition (Table 2). The seed mass did not

170 vary significantly with pollination treatment (ANOVA $F_{6,37}=1.17, P=0.34$ ), while the

171 proportion of seeds with an embryo differed significantly among pollination treatment (ANOVA

$\left.172 F_{6,37}=2.43, P=0.04\right)$. In general, the pollination experiments indicated that outcrossing tended

173 to increase both seed mass and the number of seeds with embryo, suggesting a negative impact

174 of self-pollination, although the differences were not always significant (Table 2).

175 The observation of floral morphology confirmed that most of the hermaphroditic flowers

176 possessed an effective self-pollination system, in which the rostellum was poorly developed,

177 allowing contact between the stigma and pollinaria (Fig. 2B), whereas the others had functional

178 rostella and were therefore unlikely to be autogamous (Fig. 2D). The female flowers had a

179 column with neither a rostellum nor anther cap and pollinaria (Fig. 2F).

180

181 Discussion

182 Most orchid species exhibit an extreme case of hermaphroditism, owing to the fusion of male 183 and female organs into a gynostemium. Here I showed that a Japanese population of Eulophia 
184 zollingeri develops both female and hermaphroditic flowers co-occurring within the same 185 inflorescence (i.e., gynomonoecy), while Catasetum and Cycnoches typically produces unisexual 186 individuals (i.e., dioecy, Romero \& Nelson, 1986), and Satyrium ciliatum produces both 187 hermaphroditic and female individuals (i.e., gynodioecy; Huang et al., 2009). Therefore, the present study represents the first example of gynomonoecy within the Orchidaceae. However, it should be noted that gynomonoecy must not be considered a universal strategy within the species as a whole, since it was not reported in the Chinese study (Zhang et al. 2014). In this sense, it differs from the fixed systems in Catasetum, Cycnoches, and Satyrium. The hermaphroditic flowers of a Japanese E. zollingeri population often possess an effective self-pollination system, while the female flowers without agamospermy can improve the probability of outcrossing (but selfing may still occur via geitonogamous pollinations). While female flowers are generally smaller than hermaphroditic flowers in other gynomonoecious species (reviewed by Mamut et al., 2014), the size of floral parts did not differ significantly between female and hermaphroditic flowers of E. zollingeri. In addition, female flowers tend to be on the lower part of the inflorescence, suggesting that production of female flowers is not a result of resource competition. In summary, the system observed in E. zollingeri is consistent with the outcrossingbenefit hypothesis for gynomonoecy (Mamut et al., 2014).

Many models predict that plants evolve toward either complete self-fertilization or complete outcrossing (Charlesworth \& Charlesworth, 1990). However, it seems that mixedmating systems are more common in nature (Vogler \& Kalisz, 2001; Whitehead et al., 2018), possibly because mixed mating can reduce the probability of inbreeding depression via outcrossing, while still providing reproductive assurance via selfing (Goodwillie \& Weber, 2018). As such, mixed mating systems are often referred to as "best-of-both-worlds" mating systems (Davis \& Delph, 2005; Goodwillie \& Weber, 2018). Mixed mating can be accomplished by delayed selfing, occurring after all other opportunities for outcrossing have been missed, because it provides reproductive assurance without limiting outcrossing opportunities. In addition, mixed mating can also occur in species that produce two flower types within the same plant. Gynomonoecy is one of the systems involving two flower types that allows for mixed mating. Indeed, in the E. zollingeri populations investigated here, hermaphroditic flowers conferred reproductive assurance under pollinator-limited conditions, whereas female flowers, 
214 despite their susceptibility to pollen limitation, can facilitate outcrossing, because of the lack of 215 autonomous selfing (Table 2).

216 It is possible that geitonogamy reduces the possibility of outcrossing in female flowers. In 217 E. zollingeri, though, the level of geitonogamy will be low, because only a few flowers on each 218 plant are open at one time. In particular, the risk of geitonogamy is probably negligible in 219 nectarless E. zollingeri, given that pollinators are likely to quickly leave inflorescences in food220 deceptive plants (Zhang et al., 2014, 2019; Suetsugu et al., 2015). Indeed, the avoidance of 221 geitonogamy has been hypothesized as a driving force for the evolution of food deceptive 222 pollination in plants (Johnson, Peter \& Agren, 2004). Moreover, it is noteworthy that female 223 flowers tended to be on the lower part of the inflorescence, given that E. zollingeri were 224 exclusively pollinated by the halictid bee Nomia viridicinctula in China (Zhang et al., 2014) and 225 226 that bees usually visit bottom flowers first and move upwards within an inflorescence (e.g. Iwata et al., 2012). In fact, several studies have shown that pollinator behaviors lead to directional pollen flow within inflorescences and influence floral sex allocation (Brunet \& Charlesworth, 1995). The first flowers visited will receive more pollen grains from other plants, while the last flowers visited before pollinators leave the inflorescence tend to receive geitonogamous pollination but successfully export pollen grains to other plants (Kudo, Maeda \& Narita, 2001). Therefore, it has been predicted that female-biased allocation to lower flowers and male-biased allocation to those in upper positions occurs in bee-pollinated plants (Kudo, Maeda \& Narita, 2001). The variations in floral sex allocation within E. zollingeri are consistent with the theory and are probably effective for lowering the risk of geitonogamy. somewhat influenced by the degree of inbreeding depression and pollinator availability (Smithson, 2006). In E. zollingeri, pollinator-mediated fruit set was arguably low, at least in the investigated population, given that (i) direct pollinator observation was unsuccessful and (ii) pollination experiments showed that natural pollination in female flowers was recorded only in one flower. Nonetheless, a small degree of outcrossing can result in a rapid decline in linkage

241 disequilibrium across the genome and can be sufficient to overcome negative effects such as the 242 accumulation of deleterious mutations and the slowdown in adaptation rate (Culley \& Klooster, 243 2007). In addition, although the differences were not obvious (Tremblay et al. 2005), both 244 artificial allogamous pollination and natural pollination in a female flower tended to increase 
245 seed mass and the proportion of seed with an embryo in E. zollingeri (Table 2), probably 246 providing some support for the negative effect of autonomous selfing. It should be noted that, 247 while seed mass and presence of an embryo was measured as the indicator of inbreeding 248 depression, it can even under-estimate the level of inbreeding depression. Inbreeding depression 249 might be more prominent during later stages such as seed germination or seedling growth 250 (Smithson, 2006). This possibility warrants further investigation.

251 The outcrossing opportunity might be particularly important in mycoheterotrophic plants 252 exploiting their mycorrhizal partners (Suetsugu et al., 2017), given that they usually occur in 253 shaded understorey habitats with a paucity of pollinators, and that the Red Queen hypothesis 254 argues that outcrossing is maintained by antagonistic interactions between a host and a parasite 255 (Ladle, 1992; Gibson \& Fuentes, 2015). Because mycoheterotrophic plants occur mainly in 256 pollinator-hostile shaded understorey habitats, they tend to experience strong pollinator limitation, 257 unless they possess autonomous selfing ability (Klooster \& Culley, 2009; Hentrich, Kaiser \& 258 Gottsberger, 2010; Suetsugu, 2013a, 2015). In fact, the Chinese populations without autogamous 259 ability exhibited a significant difference in fruit-set between forest edge and forest populations 260 (Zhang et al., 2014). Therefore, pollination limitation due to its mycoheterotrophic habit could 26 be a driving force in the autonomous self-pollination in E. zollingeri. Consequently, most studies

262 highlighted the importance of autonomous self-pollination in mycoheterotrophic plants (Leake,

263 1994; Zhou et al., 2012; Suetsugu, 2013a, 2015). However, several recent studies have shown that 264 mixed mating systems such as outcrossing pollinators with delayed self-pollination occur in 265 mycoheterotrophic species belonging to Ericaceae and Gentianaceae, which evolved 266 mycoheterotrophy independently from E. zollingeri (Klooster and Culley, 2009; Hentrich et al., 267 2010). The mixed mating systems, including gynomonoecy, might be more common and important 268 in mycoheterotrophic plants than previously thought.

269 Overall, it can be concluded that the Japanese population of E. zollingeri studied here 270 preserve reproductive assurance by producing autonomously selfing hermaphroditic flowers and 271 still maintain the potential benefit of producing outcrossed offspring by developing female 272 flowers. In addition, while I did not conduct the pollination experiments, several other Japanese 273 populations such as Okinawa ones exhibit very similar floral morphology (Fig. S1), suggesting 274 that the strategy might be widespread at least in Japan. However, intriguingly, the Chinese 275 populations appear to develop hermaphroditic flowers that are completely dependent on bee 
276 pollinators (Zhang et al., 2014). In fact, it is well-known that plant mating systems often vary 277 widely among populations (Suetsugu, 2013b; Whitehead et al., 2018). Variations in mating 278 systems between populations usually reflect the influence of ecological factors such as the 279 availability and abundance of suitable pollinator (Suetsugu, 2013b; Schouppe et al., 2017; 280 Whitehead et al., 2018). Therefore, it is worth clarifying how common gynomonoecy with 281 autonomous selfing hermaphroditic flowers and putatively outcrossing female flowers, is across

282 the distribution range and whether the strategy is be more prevalent where its effective pollinator 283 is less abundant.

284 It is also notable that current understanding of the adaptive advantages of gynomonoecy is 285 largely limited to the Asteraceae (Marshall \& Abbott, 1984; Bertin \& Kerwin, 1998; Bertin, 286 Connors \& Kleinman, 2010; Zhang, Xie \& Du, 2012). The outcrossing hypothesis of 287 gynomonoecy has been questioned in many asteraceous taxa, given that most Asteraceae species 288 are self-incompatible (Bertin \& Kerwin, 1998). However, it has been shown that hermaphroditic 289 flowers promote seed quantity in that they are more attractive to pollinators and/or are capable of 290 autonomous selfing, while female flowers compensate for loss of male function through 291 outcrossing in non-asteraceous taxa [i.e., Silene noctiflora (Caryophyllaceae) and Eremurus 292 anisopterus (Xanthorrhoeaceae)] (Davis \& Delph, 2005; Mamut et al., 2014). Taken together 293 with these recent finding, I suggest that the ability of female flowers to reduce geitonogamy and 294 enhance outcrossing may be widespread in gynomonoecious plants. However, it should be noted 295 that although many orchids are (at least partially) parasitic on their mycorrhizal fungi and exhibit 296 strong pollinator-limitation (Leake, 1994), gynomonoecy is not prevalent within the orchid 297 family as a whole. Given that (i) hypotheses regarding the adaptive significance of gynomonoecy 298 are not mutually exclusive and (ii) the seed-feeding fly Japanagromyza tokunagai have probably 299 substantial negative impact on the reproduction of E. zollingeri (Suetsugu \& Mita, 2019), 300 benefits other than outcrossing, such as herbivory reduction, could also have contributed to the 301 evolution of gynomonoecy. Therefore, further investigation is needed to elucidate the potentially 302 diverse adaptive significance, disadvantages, and developmental constraints of gynomonoecy. 303

\section{Acknowledgements}


305 The author thanks Drs. David Roberts, Florian Schiestl, James Ackerman and an anonymous

306 reviewer for their constructive comments on earlier versions of the manuscript. I also thank

307 Nobuyuki Inoue and Tadashi Minamitani for help with the field study.

308

309 References

310 Barrett SC. 2010. Understanding plant reproductive diversity. Philosophical Transactions of the Royal Society B: Biological Sciences 365:99-109.

312

313

314

315

316

317

318

319

320

321

322

323

324

325

326

327

328

329

330

331

332

333

334

335

Bertin RI, Connors DB, Kleinman HM. 2010. Differential herbivory on disk and ray flowers of gynomonoecious asters and goldenrods (Asteraceae). Biological Journal of the Linnean Society 101:544-552.

Bertin RI, Kerwin MA. 1998. Floral sex ratios and gynomonoecy in Aster (Asteraceae). American Journal of Botany 85:235-244.

Brunet J, Charlesworth D. 1995. Floral sex allocation in sequentially blooming plants. Evolution 49:70-79.

Charlesworth D, Charlesworth B. 1990. Inbreeding depression with heterozygote advantage and its effect on selection for modifiers changing the outcrossing rate. Evolution 44:870888.

Charnov EL, Bull J. 1977. When is sex environmentally determined? Nature 266:828-830.

Christenhusz MJM, Byng JW. 2016. The number of known plants species in the world and its annual increase. Phytotaxa 261:201-217.

Culley TM, Klooster MR. 2007. The cleistogamous breeding system: A review of its frequency, evolution, and ecology in angiosperms. Botanical Review 73:1-30.

Davis SL, Delph LF. 2005. Prior selfing and gynomonoecy in Silene noctiflora L. (Caryophyllaceae): Opportunities for enhanced outcrossing and reproductive assurance. International Journal of Plant Sciences 166:475-480.

Gibson AK, Fuentes JA. 2015. A phylogenetic test of the Red Queen Hypothesis: outcrossing and parasitism in the nematode phylum. Evolution 69:530-540.

Goodwillie C, Weber JJ. 2018. The best of both worlds? A review of delayed selfing in flowering plants. American Journal of Botany 105:641-655.

Hentrich H, Kaiser R, Gottsberger G. 2010. The reproductive biology of Voyria (Gentianaceae) species in French Guiana. Taxon 59:867-880. 
336 Huang SQ, Lu Y, Chen YZ, Luo YB, Delph LF. 2009. Parthenogenesis maintains male

337

338

339

340

341

342

343

344

345

346

347

348

349

350

351

352

353

354

355

356

357

358

359

360

361

362

363

364

365

366 sterility in a gynodioecious orchid. American Naturalist 174:578-584.

Inoue K. 1986. Experimental studies on male and female reproductive success: effects of variation in spur length and pollinator activity on Platanthera mandarinorum ssp. hachijoensis (Orchidaceae). Plant Species Biology 1:207-215.

Iwata T, Nagasaki O, Ishii HS, Ushimaru A. 2012. Inflorescence architecture affects pollinator behaviour and mating success in Spiranthes sinensis (Orchidaceae). New Phytologist 193: 196-203.

Johnson SD, Peter CI, Ågren J. 2004. The effects of nectar addition on pollen removal and geitonogamy in the non-rewarding orchid Anacamptis morio. Proceedings of the Royal Society of London B: Biological Sciences 271:803-809.

Klooster MR, Culley TM. 2009. Comparative analysis of the reproductive ecology of Monotropa and Monotropsis: Two mycoheterotrophic genera in the Monotropoideae (Ericaceae). American Journal of Botany 96:1337-1347.

Kudo G, Maeda T, Narita K. 2001. Variation in floral sex allocation and reproductive success within inflorescences of Corydalis ambigua (Fumariaceae): Pollination efficiency or resource limitation? Journal of Ecology 89:48-56.

Ladle RJ. 1992. Parasites and sex: Catching the red queen. Trends in Ecology and Evolution 7:405-408.

Leake JR. 1994. The biology of myco-heterotrophic ('saprophytic') plants. New Phytologist 127:171-216.

Lloyd DG. 1979. Parental strategies of angiosperms. New Zealand Journal of Botany 17:595606.

Lu Y, Huang SQ. 2006. Adaptive advantages of gynomonoecious species. Acta Phytotaxonomica Sinica 44:231-239.

Mamut J, Xiong YZ, Tan DY, Huang SQ. 2014. Pistillate flowers experience more pollen limitation and less geitonogamy than perfect flowers in a gynomonoecious herb. New Phytologist 201:670-677.

Marshall DF, Abbott RJ. 1984. Polymorphism for outcrossing frequency at the ray floret locus in Senecio vulgaris L. III. Causes. Heredity 53:145-149.

Nilsson LA. 1988. The evolution of flowers with deep corolla tubes. Nature 334:147-149. 
367

368

369

370

371

372

373

374

375

376

377

378

379

380

381

382

383

384

385

386

387

388

389

390

391

392

393

394

395

396

397

Ogura-Tsujita Y, Yukawa T. 2008. High mycorrhizal specificity in a widespread mycoheterotrophic plant, Eulophia zollingeri (Orchidaceae). American Journal of Botany 95:93-97.

Pannell JR. 2009. Mating-system evolution: succeeding by celibacy. Current Biology 19:R983R985.

Romero GA. 1992. Non-functional flowers in Catasetum orchids (Catasetinae, Orchidaceae). Botanical Journal of the Linnean Society 109:305-313.

Romero GA, Nelson CE. 1986. Sexual dimorphism in Catasetum orchids: Forcible pollen emplacement and male flower competition. Science 232:1538-1540.

Rudall PJ, Bateman RM. 2002. Roles of synorganisation, zygomorphy and heterotopy in floral evolution: The gynostemium and labellum of orchids and other lilioid monocots. Biological reviews of the Cambridge Philosophical Society 77:403-441.

Schouppe D, Brys R, Vallejo-Marin M, Jacquemyn H. 2017. Geographic variation in floral traits and the capacity of autonomous selfing across allopatric and sympatric populations of two closely related Centaurium species. Scientific Reports 7:46410.

Sletvold N, Gren JA. 2011. Nonadditive effects of floral display and spur length on reproductive success in a deceptive orchid. Ecology 92:2167-2174.

Smithson A. 2006. Pollinator limitation and inbreeding depression in orchid species with and without nectar rewards. New Phytologist 169:419-430.

Suetsugu K. 2013a. Autogamous fruit set in a mycoheterotrophic orchid Cyrtosia septentrionalis. Plant Systematics and Evolution 299:481-486.

Suetsugu K. 2013. Delayed autonomous self-pollination in two Japanese varieties of Epipactis helleborine (Orchidaceae). Botanical Journal of the Linnean Society 173:733-743.

Suetsugu K. 2015. Autonomous self-pollination and insect visitors in partially and fully mycoheterotrophic species of Cymbidium (Orchidaceae). Journal of Plant Research 128:115-125.

Suetsugu K, Mita T. 2019. First report that the wasp Gronotoma guamensis (Hymenoptera: Figitidae: Eucoilinae) parasitizes the orchid-feeding fly Japanagromyza tokunagai in Japan. Entomological Science 22:194-197.

Suetsugu K, Matsubayashi J, Tayasu I. 2020. Some mycoheterotrophic orchids depend on carbon from dead wood: Novel evidence from a radiocarbon approach. New Phytologist 
398

399

400

401

402

403

404

405

406

407

408

409

410

411

412

413

414

415

416

417

418

419

420

421

422

423

424

425

426

427

428

227:1519-1529.

Suetsugu K, Naito RS, Fukushima S, Kawakita A, Kato M. 2015. Pollination system and the effect of inflorescence size on fruit set in the deceptive orchid Cephalanthera falcata. Journal of Plant Research 128:585-594.

\section{Suetsugu K, Yamato M, Miura C, Yamaguchi K, Takahashi K, Ida Y, Shigenobu S,} Kaminaka H. 2017. Comparison of green and albino individuals of the partially mycoheterotrophic orchid Epipactis helleborine on molecular identities of mycorrhizal fungi, nutritional modes and gene expression in mycorrhizal roots. Molecular Ecology 26:1652-1669.

Tremblay RL, Ackerman JD, Zimmerman JK, Calvo RN. 2005. Variation in sexual reproduction in orchids and its evolutionary consequences: A spasmodic journey to diversification. Biological Journal of the Linnean Society 84:1-54.

Vogler DW, Kalisz S. 2001. Sex among the flowers: The distribution of plant mating systems. Evolution 55:202-204.

Whitehead MR, Lanfear R, Mitchell RJ, Karron JD. 2018. Plant mating systems often vary widely among populations. Frontiers in Ecology and Evolution 6:38.

Yampolsky C, Yampolsky H. 1922. Distribution of sex forms in the phanerogamic flora. Bibliotheca Genetica 3:1-62.

Zhang G, Xie T, Du G. 2012. Variation in floral sex allocation, female success, and seed predation within racemiform synflorescence in the gynomonoecious Ligularia virgaurea (Asteraceae). Journal of Plant Research 125:527-538.

Zhang ZB, Yang M, Zhao XH, Ni SD, Yang FP, Chen QQ, Huang BG. 2014. Deceptive pollination of a saprophytic orchid, Eulophia zollingeri. Guangxi Zhiwu/Guihaia 34:541547.

Zhang Z, Gale SW, Li JH, Fischer GA, Ren MX, Song XQ. 2019. Pollen-mediated gene flow ensures connectivity among spatially discrete sub-populations of Phalaenopsis pulcherrima, a tropical food-deceptive orchid. BMC Plant Biology 19:597.

Zhou X, Lin H, Fan XL, Gao JY. 2012. Autonomous self-pollination and insect visitation in a saprophytic orchid, Epipogium roseum (D.Don) Lindl. Australian Journal of Botany 60:154159. 


\section{Figure legends}

430

431 Figure 1 Eulophia zollingeri in its natural habitat. (A) Inflorescence. (B) Hermaphroditic 432 flower. (C) Female flower.

433

434 Figure 2 Column morphology of Eulophia zollingeri flowers. (A, B) Column with a 435 degenerate rostellum, which facilitates autogamy. (C, D) Column with a well-developed 436 rostellum, which prevents autogamy. (E, F) Column with neither a rostellum nor anther cap and 437 pollinaria. AC, anther cap; RS, rostellum; PO, pollinaria; ST, stigma. 


\section{Figure 1}

Eulophia zollingeri in its natural habitat

Eulophia zollingeri in its natural habitat. (A) Inflorescence. (B) Hermaphroditic flower.

(C) Female flower.
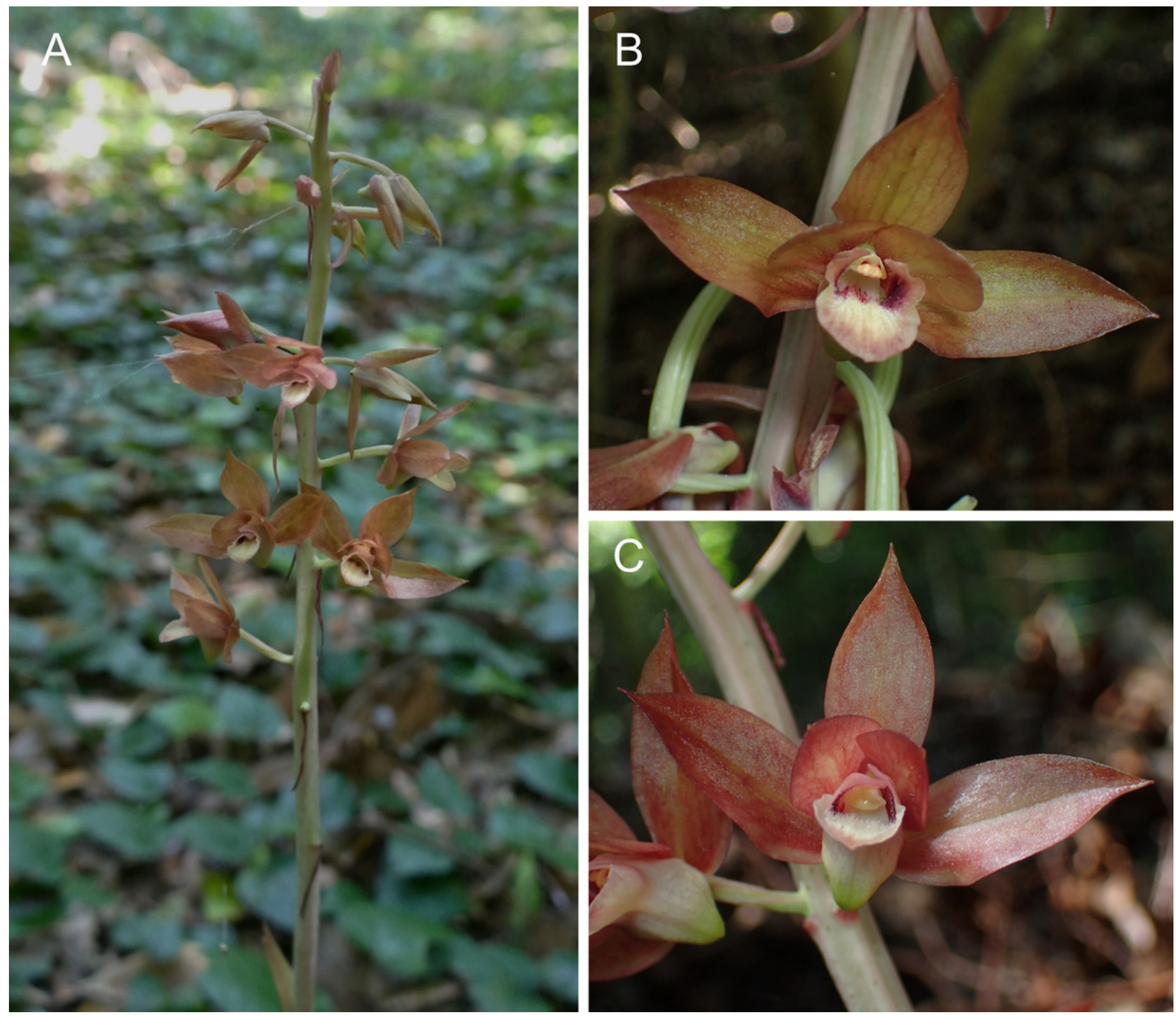
Figure 2

Column morphology of Eulophia zollingeri flowers.

Column morphology of Eulophia zollingeri flowers. (A, B) Column with a degenerate rostellum, which facilitates autogamy. (C, D) Column with a well-developed rostellum, which prevents autogamy. (E, F) Column with neither a rostellum nor anther cap and pollinaria. AC, anther cap; RS, rostellum; PO, pollinaria, ST, stigma. 

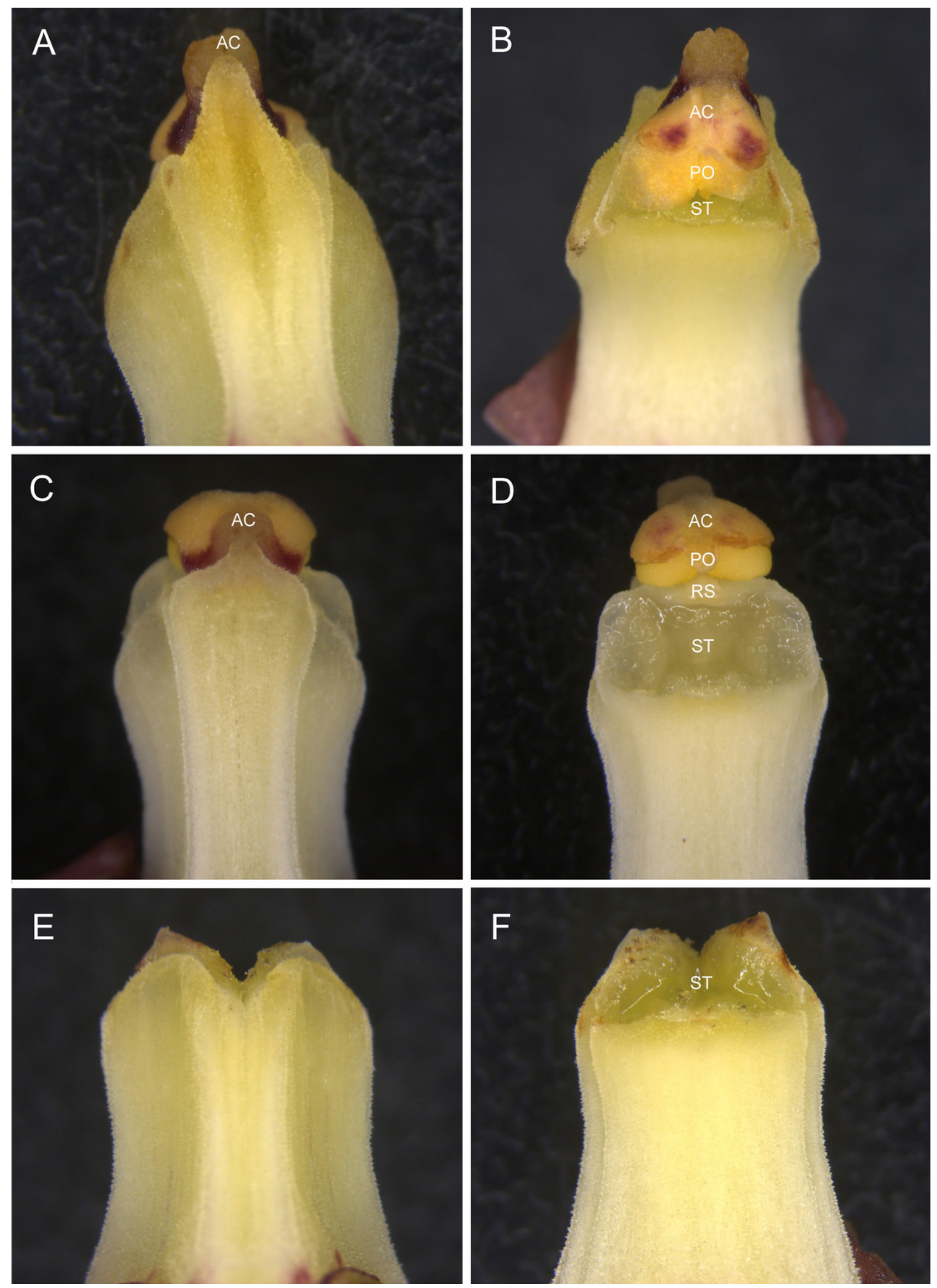

PeerJ reviewing PDF | (2020:07:51078:2:1:NEW 26 Sep 2020) 


\section{Table $\mathbf{1}$ (on next page)}

Comparison of the length of the dorsal sepal, lateral sepal, lateral petal, and lip, between hermaphroditic and female flowers in Eulophia zollingeri 
Table 1. Comparison of the length of the dorsal sepal, lateral sepal, lateral petal, and lip, between hermaphroditic and female flowers in Eulophia zollingeri.

\begin{tabular}{lllll}
\hline Flower type & Dorsal sepal $(\mathrm{mm})$ & Lateral sepal $(\mathrm{mm})$ & Lateral petal $(\mathrm{mm})$ & Lip $(\mathrm{mm})$ \\
\hline Hermaphroditic & $23.5 \pm 1.8$ & $25.6 \pm 2.1$ & $17.8 \pm 1.5$ & $19.7 \pm 0.7$ \\
Female & $24.8 \pm 1.6$ & $26.2 \pm 1.8$ & $18.2 \pm 1.3$ & $20.0 \pm 0.9$ \\
\hline
\end{tabular}

The lengths are expressed by mean $\pm \mathrm{SD}(\mathrm{mm})$.

1 


\section{Table 2 (on next page)}

Effect of pollination treatment on fruit set, seed mass, and proportion of seeds with embryo in Eulophia zollingeri 
Table 2. Effect of pollination treatment on fruit set, seed mass, and proportion of seeds with an embryo in Eulophia zollingeri.

\begin{tabular}{|c|c|c|c|c|c|}
\hline Flower type & Treatment & $\begin{array}{l}\text { Manual } \\
\text { allogamy }\end{array}$ & $\begin{array}{l}\text { Manual } \\
\text { geitonogamy }\end{array}$ & $\begin{array}{l}\text { Autonomous } \\
\text { autogamy }\end{array}$ & Open \\
\hline \multirow[t]{3}{*}{ Hermaphroditic } & Fruit set (\%) & $70.0^{\mathrm{a}}$ & $70.0^{\mathrm{a}}$ & $50.0^{\mathrm{a}}$ & $57.1^{\mathrm{a}}$ \\
\hline & Seed mass & $31.6 \pm 11.6$ & $24.1 \pm 11.4$ & $20.8 \pm 9.3$ & $24.9 \pm 8.6$ \\
\hline & $\begin{array}{l}\text { Seeds with } \\
\text { embryo }\end{array}$ & $82.4 \pm 4.2^{\mathrm{ac}}$ & $79.0 \pm 4.2^{\mathrm{abc}}$ & $77.2 \pm 2.9^{\mathrm{bc}}$ & $79.2 \pm 3.6^{\mathrm{c}}$ \\
\hline \multirow[t]{3}{*}{ Female } & Fruit set (\%) & $60.0^{\mathrm{a}}$ & $60.0^{\mathrm{a}}$ & 0.0 & $5.3^{\mathrm{b}}$ \\
\hline & Seed mass & $30.2 \pm 14.7$ & $25.7 \pm 11.0$ & - & 44.9 \\
\hline & $\begin{array}{l}\text { Seeds with } \\
\text { embryo }\end{array}$ & $83.3 \pm 4.4^{\mathrm{a}}$ & $77.3 \pm 2.9^{\mathrm{bc}}$ & - & $82.0^{\mathrm{abc}}$ \\
\hline
\end{tabular}

Different superscript letters indicate significant differences $(P<0.05)$ between treatment groups. Both seed mass and seeds with an embryo are expressed by mean $\pm \mathrm{SD}$, whenever the sample size is more than $>1$. 